KYUNGPOOK Math. J. 48(2008), 15-24

\title{
Kato's Inequalities for Degenerate Quasilinear Elliptic Operators
}

Dedicated to Prof. M. Nagase

TOSHIO HORIUCHI

Department of Mathematical Science, Ibaraki University Mito, Ibaraki, 310, Japan. e-mail : horiuchi@mx.ibaraki.ac.jp

Abstract. Let $N \geq 1$ and $p>1$. Let $\Omega$ be a domain of $\mathbb{R}^{N}$. In this article we shall establish Kato's inequalities for quasilinear degenerate elliptic operators of the form $\mathcal{A}_{p} u=\operatorname{div} A(x, \nabla u)$ for $u \in K_{p}(\Omega)$, where $K_{p}(\Omega)$ is an admissible class and $A(x, \xi): \Omega \times \mathbb{R}^{N} \rightarrow \mathbb{R}^{N}$ is a mapping satisfying some structural conditions. If $p=2$ for example, then we have $K_{2}(\Omega)=\left\{u \in L_{l o c}^{1}(\Omega): \partial_{j} u, \partial_{j, k}^{2} u \in L_{l o c}^{1}(\Omega)\right.$ for $\left.j, k=1,2, \cdots, N\right\}$. Then we shall prove that $\mathcal{A}_{p}|u| \geq(\operatorname{sgn} u) \mathcal{A}_{p} u$ and $\mathcal{A}_{p} u^{+} \geq\left(\operatorname{sgn}^{+} u\right)^{p-1} \mathcal{A}_{p} u$ in $\mathcal{D}^{\prime}(\Omega)$ with $u \in K_{p}(\Omega)$. These inequalities are called Kato's inequalities provided that $p=2$. The class of operators $\mathcal{A}_{p}$ contains the so-called $p$-harmonic operators $L_{p}=\operatorname{div}\left(|\nabla u|^{p-2} \nabla u\right)$ for $A(x, \xi)=|\xi|^{p-2} \xi$.

\section{Introduction}

Let $N \geq 1$ and $p>1$. Let $\Omega$ be a domain of $\mathbb{R}^{N}$. In this paper we shall consider the operators of the form

$$
\mathcal{A}_{p} u=\operatorname{div} A(x, \nabla u),
$$

where $A: \Omega \times \mathbb{R}^{N} \rightarrow \mathbb{R}^{N}$ is a mapping satisfying the following conditions for some positive constants $C_{1}, C_{2}$ and $C_{3}$ :

$$
\begin{gathered}
A(x, \xi) \in C^{1}\left(\Omega \times\left(\mathbb{R}^{N} \backslash\{0\}\right) ; \mathbb{R}^{N}\right), \\
A(x, \xi) \cdot \xi \geq C_{1}|\xi|^{p}, \\
|A(x, \xi)| \leq C_{2}|\xi|^{p-1}, \\
\left|\nabla_{x} A(x, \xi)\right| \leq C_{3}|\xi|^{p-1}, \quad\left|\nabla_{\xi} A(x, \xi)\right| \leq C_{3}|\xi|^{p-2},
\end{gathered}
$$

Received June 13, 2006, and in revised form October 16, 2006. 2000 Mathematics Subject Classification: 35J70, 35J60.

Key words and phrases: Kato's inequality, $p$-harmonic operators. 
whenever $x \in \Omega$ and $\xi \in \mathbb{R}^{N} \backslash\{0\}$; and

$$
A(x, \lambda \xi)=\lambda|\lambda|^{p-2} A(x, \xi),
$$

whenever $x \in \Omega, \xi \in \mathbb{R}^{N} \backslash\{0\}$ and $\lambda \in \mathbb{R}$.

When $p \in(1,2)$, we further assume that $A(x, \xi)$ has the form

$$
A(x, \xi)=a(x,|\xi|) \xi,
$$

where $a(x, r) \in C^{1}\left(\Omega \times \mathbb{R}_{+} ; \mathbb{R}_{+}\right)$, satisfying the condition

$$
a(x, r)+r \frac{\partial}{\partial r} a(x, r) \geq 0,
$$

whenever $r \in \mathbb{R}_{+}$. From the previous assumptions (1.3) and (1.4) we see that $a(x,|\xi|)$ satisfies

$$
C_{1}|\xi|^{p-2} \leq a(x,|\xi|) \leq C_{2}|\xi|^{p-2},
$$

whenever $\xi \in \mathbb{R}^{N} \backslash\{0\}$; and

$$
a(x,|\lambda \xi|)=|\lambda|^{p-2} a(x,|\xi|),
$$

whenever $\lambda \in \mathbb{R}, \lambda \neq 0$.

We also define the $p$-harmonic operator $L_{p}$ in the following way:

$$
\begin{aligned}
L_{p} u & =\operatorname{div}\left(|\nabla u|^{p-2} \nabla u\right) \\
& =|\nabla u|^{p-2} \Delta u+(p-2)|\nabla u|^{p-4} \sum_{j, k=1}^{N} \partial_{j} u \partial_{k} u \partial_{j, k}^{2} u
\end{aligned}
$$

where $p>1$ and $\partial_{j} u=\frac{\partial u}{\partial x_{j}}, \partial_{j, k}^{2} u=\frac{\partial^{2} u}{\partial x_{j} \partial x_{k}}$ for $j, k=1,2, \cdots, N$. Then it is clear that we obtain $L_{p}$ as a special case for $A(x, \xi)=|\xi|^{p-2} \xi\left(\right.$ or $a(x,|\xi|)=|\xi|^{p-2}$ ).

In this paper we shall establish so-called Kato's inequalities for the operators $\mathcal{A}_{p} u=\operatorname{div} A(x, \nabla u)$ in place of linear elliptic operators represented by the Laplacian $\Delta$. In the rest of this section we recall well-known Kato's inequalities. Define

$$
M\left(x, \partial_{x}\right)=\partial_{j}\left(a_{j k}(x) \partial_{k} \cdot\right),
$$

where $a_{j k}(x) \in C^{1}(\Omega)$ is positive definite in the following sense.

$$
\sum_{j, k=1}^{N} a_{j k}(x) \xi_{j} \xi_{k} \geq C|\xi|^{2}, \quad \text { for any } \xi \in \mathbb{R}^{N} \backslash\{0\} \text { and } x \in \Omega .
$$


Here $C$ is a positive number independent of each $x$ and $\xi$. Then we have the following Kato's inequalities. (For the proof, see [1]).

Theorem 1.1. For $u$ and $M\left(x, \partial_{x}\right) u \in L_{l o c}^{1}(\Omega)$, we have

$$
\begin{array}{ll}
M\left(x, \partial_{x}\right)|u| \geq\left(M\left(x, \partial_{x}\right) u\right) \operatorname{sgn} u & \text { in } \mathcal{D}^{\prime}(\Omega), \\
M\left(x, \partial_{x}\right) u_{+} \geq\left(M\left(x, \partial_{x}\right) u\right) \operatorname{sgn}^{+} u & \text { in } \mathcal{D}^{\prime}(\Omega) .
\end{array}
$$

Here

$$
\operatorname{sgn} u(x)=\left\{\begin{array}{l}
\frac{u(x)}{|u(x)|}, \text { for } u \neq 0, \\
0, \quad \text { for } u=0 .
\end{array} \quad \operatorname{sgn}^{+} u(x)=\left\{\begin{array}{l}
1, \text { for } u>0, \\
1 / 2, \text { for } u=0, \\
0, \text { for } u<0,
\end{array}\right.\right.
$$

and $u_{+}=\max [u(x), 0]$. By $\mathcal{D}^{\prime}(\Omega)$ we denote the set of all distributions on $\Omega$.

This paper is organized in the following way. In $\S 2$ we prepare basic inequalities including the operators $\mathcal{A}_{p}$. In $\S 3$ we shall state our main result, and the proof is also given there.

\section{Preliminary}

We shall establish some fundamental inequalities for smooth functions $u$, which are useful to prove our main result.

Lemma 2.1. Assume that $u \in C^{2}(\Omega)$. Then it holds that

$$
\begin{cases}\mathcal{A}_{p}|u| \geq(\operatorname{sgn} u) \mathcal{A}_{p} u & \text { in } \mathcal{D}^{\prime}(\Omega), \\ \mathcal{A}_{p} u_{+} \geq\left(\operatorname{sgn}^{+} u\right)^{p-1} \mathcal{A}_{p} u & \text { in } \mathcal{D}^{\prime}(\Omega) .\end{cases}
$$

Here by $\mathcal{D}^{\prime}(\Omega)$ we denote the set of all distributions on $\Omega$.

Proof. For any $\varepsilon>0$ we set

$$
u_{\varepsilon}=\left(u^{2}+\varepsilon^{2}\right)^{1 / 2}
$$

Then we see

$$
\begin{aligned}
\partial_{j} u_{\varepsilon} & =\frac{u}{u_{\varepsilon}} \partial_{j} u \\
\partial_{j}^{2} u_{\varepsilon} & =\frac{u}{u_{\varepsilon}} \partial_{j}^{2} u+\frac{1}{u_{\varepsilon}}\left(1-\left(\frac{u}{u_{\varepsilon}}\right)^{2}\right)\left(\partial_{j} u\right)^{2} \geq \frac{u}{u_{\varepsilon}} \partial_{j}^{2} u
\end{aligned}
$$

Here $\partial_{j}^{2} u=\frac{\partial^{2} u}{\partial x_{j}^{2}}, j=1,2, \cdots, N$. Using these we have

$$
\begin{aligned}
\mathcal{A}_{p} u_{\varepsilon} & =\left|\frac{u}{u_{\varepsilon}}\right|^{p-2}\left(\frac{u}{u_{\varepsilon}} \mathcal{A}_{p} u+(p-1) \frac{1}{u_{\varepsilon}}\left(1-\left(\frac{u}{u_{\varepsilon}}\right)^{2}\right) A(x, \nabla u) \cdot \nabla u\right) \\
& \geq\left|\frac{u}{u_{\varepsilon}}\right|^{p-2} \frac{u}{u_{\varepsilon}} \mathcal{A}_{p} u
\end{aligned}
$$


In a similar way we have

$$
\begin{aligned}
& \mathcal{A}_{p}\left(\frac{u+u_{\varepsilon}}{2}\right) \\
= & \left(\frac{1+\frac{u}{u_{\varepsilon}}}{2}\right)^{p-1}\left(\mathcal{A}_{p} u+(p-1) \frac{1}{u_{\varepsilon}}\left(1-\frac{u}{u_{\varepsilon}}\right) A(x, \nabla u) \cdot \nabla u\right) \\
\geq & \left(\frac{1+\frac{u}{u_{\varepsilon}}}{2}\right)^{p-1} \mathcal{A}_{p} u
\end{aligned}
$$

Since $2 u_{+}=u+|u|$ holds, letting $\varepsilon \rightarrow 0$ we have the desired inequalities.

When $p \in(1,2)$, we need to consider the following regularized operator. Define $\mathcal{A}_{p, \eta}$ for $\eta \geq 0$

$$
\mathcal{A}_{p, \eta} u=\operatorname{div}\left(a\left(x,\left(\eta^{2}+|\nabla u|^{2}\right)^{\frac{1}{2}}\right) \nabla u\right) .
$$

Let us set $|\nabla u|_{\eta}=\left(\eta^{2}+|\nabla u|^{2}\right)^{\frac{1}{2}}$ and $r=|\xi|$. Then we see

$$
\begin{aligned}
\mathcal{A}_{p, \eta} u_{\varepsilon}= & \frac{u}{u_{\varepsilon}}\left(a\left(x,\left|\nabla u_{\varepsilon}\right|_{\eta}\right) \Delta u+\nabla u \cdot \nabla_{x} a\left(x,\left|\nabla u_{\varepsilon}\right|_{\eta}\right)\right. \\
& \left.+\frac{\partial}{\partial r} a\left(x,\left|\nabla u_{\varepsilon}\right|_{\eta}\right)\left|\nabla u_{\varepsilon}\right|_{\eta}\left(\frac{u}{u_{\varepsilon}}\right)^{2} \sum_{j, k} \frac{\partial_{j} u \partial_{k} u \partial_{j, k}^{2} u}{\left|\nabla u_{\varepsilon}\right|_{\eta}^{2}}\right) \\
& +a\left(x,\left|\nabla u_{\varepsilon}\right|_{\eta}\right) \frac{1}{u_{\varepsilon}}\left(1-\left(\frac{u}{u_{\varepsilon}}\right)^{2}\right)|\nabla u|^{2} \\
& +\frac{\partial}{\partial r} a\left(x,\left|\nabla u_{\varepsilon}\right|_{\eta}\right)\left|\nabla u_{\varepsilon}\right|_{\eta}\left(\frac{u}{u_{\varepsilon}}\right)^{2}|\nabla u|^{2} \frac{1}{u_{\varepsilon}}\left(1-\left(\frac{u}{u_{\varepsilon}}\right)^{2}\right) \frac{|\nabla u|^{2}}{\left|\nabla u_{\varepsilon}\right|_{\eta}^{2}} \\
\geq & \frac{u}{u_{\varepsilon}}\left(a\left(x,\left|\nabla u_{\varepsilon}\right|_{\eta}\right) \Delta u+\nabla u \cdot \nabla_{x} a\left(x,\left|\nabla u_{\varepsilon}\right|_{\eta}\right)\right. \\
& \left.+\frac{\partial}{\partial r} a\left(x,\left|\nabla u_{\varepsilon}\right|_{\eta}\right)\left|\nabla u_{\varepsilon}\right|_{\eta}\left(\frac{u}{u_{\varepsilon}}\right)^{2} \sum_{j, k} \frac{\partial_{j} u \partial_{k} u \partial_{j, k}^{2} u}{\left|\nabla u_{\varepsilon}\right|_{\eta}^{2}}\right)
\end{aligned}
$$

Similarly we can compute $\mathcal{A}_{p, \eta}\left(\frac{u+u_{\varepsilon}}{2}\right)$ to obtain the following :

$$
\begin{aligned}
& \mathcal{A}_{p, \eta}\left(\frac{u+u_{\varepsilon}}{2}\right) \\
= & w_{\varepsilon}\left(a\left(x,|\tilde{\nabla} u|_{\eta}\right) \Delta u+\nabla u \cdot \nabla_{x} a\left(x,|\tilde{\nabla} u|_{\eta}\right)\right. \\
& \left.+\frac{\partial}{\partial r} a\left(x,|\tilde{\nabla} u|_{\eta}\right)|\tilde{\nabla} u|_{\eta} w_{\varepsilon}^{2} \sum_{j, k} \frac{\partial_{j, k} u \partial_{j} u \partial_{k} u}{|\tilde{\nabla} u|_{\eta}^{2}}\right) \\
& +\nabla w_{\varepsilon} \cdot \nabla u\left(a\left(x,|\tilde{\nabla} u|_{\eta}\right)+\frac{\partial}{\partial r} a\left(x,|\tilde{\nabla} u|_{\eta}\right)|\tilde{\nabla} u|_{\eta} \frac{w_{\varepsilon}^{2}|\nabla u|^{2}}{|\tilde{\nabla} u|_{\eta}^{2}}\right)
\end{aligned}
$$


Here

$$
\left\{\begin{array}{l}
w_{\varepsilon}=\frac{1}{2}\left(1+\frac{u}{u_{\varepsilon}}\right) \\
\nabla w_{\varepsilon} \cdot \nabla u=\frac{w_{\varepsilon}}{u_{\varepsilon}}\left(1-\frac{u}{u_{\varepsilon}}\right)|\nabla u|^{2} \\
|\tilde{\nabla} u|_{\eta}=\left(\eta^{2}+w_{\varepsilon}^{2}|\nabla u|^{2}\right)^{\frac{1}{2}}
\end{array}\right.
$$

Then we have

$$
\begin{aligned}
\mathcal{A}_{p, \eta}\left(\frac{u+u_{\varepsilon}}{2}\right) \geq & w_{\varepsilon}\left(a\left(x,|\tilde{\nabla} u|_{\eta}\right) \Delta u+\nabla u \cdot \nabla_{x} a\left(x,|\tilde{\nabla} u|_{\eta}\right)\right. \\
& \left.+\frac{\partial}{\partial r} a\left(x,|\tilde{\nabla} u|_{\eta}\right)|\tilde{\nabla} u|_{\eta} w_{\varepsilon}^{2} \sum_{j, k} \frac{\partial_{j, k} u \partial_{j} u \partial_{k} u}{|\tilde{\nabla} u|_{\eta}^{2}}\right)
\end{aligned}
$$

Therefore we have

Lemma 2.2. For $u \in C^{2}(\Omega)$ it holds that

$$
\begin{array}{r}
\mathcal{A}_{p, \eta} u_{\varepsilon} \geq \frac{u}{u_{\varepsilon}}\left(a\left(x,\left|\nabla u_{\varepsilon}\right|_{\eta}\right) \Delta u+\nabla u \cdot \nabla_{x} a\left(x,\left|\nabla u_{\varepsilon}\right|_{\eta}\right)\right. \\
\left.+\frac{\partial}{\partial r} a\left(x,\left|\nabla u_{\varepsilon}\right|_{\eta}\right)\left|\nabla u_{\varepsilon}\right|_{\eta}\left(\frac{u}{u_{\varepsilon}}\right)^{2} \sum_{j, k} \frac{\partial_{j} u \partial_{k} u \partial_{j, k}^{2} u}{\left|\nabla u_{\varepsilon}\right|_{\eta}^{2}}\right) \\
\mathcal{A}_{p, \eta}\left(\frac{u+u_{\varepsilon}}{2}\right) \geq \\
\quad w_{\varepsilon}\left(a\left(x,|\tilde{\nabla} u|_{\eta}\right) \Delta u+\nabla u \cdot \nabla_{x} a\left(x,|\tilde{\nabla} u|_{\eta}\right)\right. \\
\left.+\frac{\partial}{\partial r} a\left(x,|\tilde{\nabla} u|_{\eta}\right)|\tilde{\nabla} u|_{\eta} w_{\varepsilon}^{2} \sum_{j, k} \frac{\partial_{j, k} u \partial_{j} u \partial_{k} u}{|\tilde{\nabla} u|_{\eta}^{2}}\right)
\end{array}
$$

Letting $\varepsilon \rightarrow 0$, we have for $u \in C^{2}(\Omega)$.

Lemma 2.3. For $u \in C^{2}(\Omega)$ it holds that in $\mathcal{D}^{\prime}(\Omega)$

$$
\begin{aligned}
\mathcal{A}_{p, \eta}|u| \geq & (\operatorname{sgn} u)\left(a\left(x,|\nabla u|_{\eta}\right) \Delta u+\nabla u \cdot \nabla_{x} a\left(x,|\nabla u|_{\eta}\right)\right. \\
& \left.+\frac{\partial}{\partial r} a\left(x,|\nabla u|_{\eta}\right)|\nabla u|_{\eta} \sum_{j, k} \frac{\partial_{j} u \partial_{k} u \partial_{j, k}^{2} u}{|\nabla u|_{\eta}^{2}}\right) \\
= & (\operatorname{sgn} u) \mathcal{A}_{p, \eta} u
\end{aligned}
$$




$$
\begin{aligned}
\mathcal{A}_{p, \eta} u_{+} \geq & \left(\operatorname{sgn}^{+} u\right)\left(a\left(x,\left(\eta^{2}+\left(\operatorname{sgn}^{+} u\right)^{2}|\nabla u|^{2}\right)^{\frac{1}{2}}\right) \Delta u\right. \\
& +\nabla u \cdot \nabla_{x} a\left(x,\left(\eta^{2}+\left(\operatorname{sgn}^{+} u\right)^{2}|\nabla u|^{2}\right)^{\frac{1}{2}}\right) \\
& +\frac{\partial}{\partial r} a\left(x,\left(\eta^{2}+\left(\operatorname{sgn}^{+} u\right)^{2}|\nabla u|^{2}\right)^{\frac{1}{2}}\right)\left(\operatorname{sgn}^{+} u\right)^{2} \\
& \left.\sum_{j, k} \frac{\partial_{j, k} u \partial_{j} u \partial_{k} u}{\left(\eta^{2}+\left(\operatorname{sgn}^{+} u\right)^{2}|\nabla u|^{2}\right)^{\frac{1}{2}}}\right)
\end{aligned}
$$

\section{Main result}

We introduce an admissible class $K_{p}(\Omega)$ for the operators $\mathcal{A}_{p}$.

Definition 3.1. Let $p>1$ and $p^{*}=\max (p-1,1)$. We set

$$
\begin{gathered}
K_{p}(\Omega)=\left\{u \in L_{l o c}^{1}(\Omega): \partial_{j} u, \partial_{j k}^{2} u \in L_{l o c}^{p^{*}}(\Omega),|\nabla u|^{p-2}\left|\partial_{j, k}^{2} u\right| \in L_{l o c}^{1}(\Omega)\right. \\
\text { for } j, k=1,2, \cdots, N\} .
\end{gathered}
$$

Now we are in a position to state our main result.

Theorem 3.1. Let $p>1$. Assume that $u \in K_{p}(\Omega)$, then it holds that in $\mathcal{D}^{\prime}(\Omega)$

$$
\left\{\begin{array}{l}
\mathcal{A}_{p}|u| \geq(\operatorname{sgn} u) \mathcal{A}_{p} u \\
\mathcal{A}_{p} u_{+} \geq\left(\operatorname{sgn}^{+} u\right)^{p-1} \mathcal{A}_{p} u .
\end{array}\right.
$$

Remark 3.2. (1) If $p=2$, then $K_{2}(\Omega)=\left\{u \in L_{l o c}^{1}(\Omega): \partial_{j} u, \partial_{j k}^{2} u \in\right.$ $L_{l o c}^{1}(\Omega)$, for $\left.j, k=1,2, \cdots, N\right\}$. Since $L_{2}=\Delta$ in this case, it is known that Kato's inequalities hold under the assumptions that $u, \Delta u \in L_{\text {loc }}^{1}(\Omega)$. But if $p \neq 2$, the operators $\mathcal{A}_{p}$ and $L_{p}$ become nonlinear. Hence it was needed to introduce the class $K_{p}$.

(2) If $p>2$ and $\partial_{j} u, \partial_{j k}^{2} u \in L_{l o c}^{p^{*}}(\Omega)$, then we see $|\nabla u|^{p-2}\left|\partial_{j k}^{2} u\right| \in L_{l o c}^{1}(\Omega)$ by a Hölder's inequality. (See the proof of Theorem 3.1.)

(3) It suffices to prove this theorem assuming that $u \in K_{p}(\Omega)$ has a compact support.

Proof. Without loss of generality, we assume that $\Omega=\mathbb{R}^{N}$ and $u \in K_{p}\left(\mathbb{R}^{N}\right)$ has a compact support. If $u \in C^{2}\left(\mathbb{R}^{N}\right)$, then the assertions follow from Lemma 2.1. Hence we approximate an integrable function $u$ by smooth functions $u_{\rho}(\rho>0)$ as follows: Let us set $B_{r}=\left\{x \in \mathbb{R}^{N}:|x|<r\right\}$. Let $\varphi \in C_{0}^{\infty}\left(\mathbb{R}^{N}\right)$ satisfy $\varphi \geq 0, \int_{\mathbb{R}^{N}} \varphi(x) d x=1$ and $\varphi=0$ in $B_{2}^{c}$. Moreover we assume that $\varphi$ is radial and decreasing. Now we set $\varphi_{\rho}(x)=\rho^{-N} \varphi(x / \rho)$ for $\rho>0$ and define

$$
u_{\rho}(x)=u * \varphi_{\rho}(x) \equiv \int_{\mathbb{R}^{N}} u(x-y) \varphi_{\rho}(y) d y .
$$


Then it is clear from the assumptions on $u$ and $\varphi$ that as $\rho \rightarrow 0$

$$
\left\{\begin{array}{l}
u_{\rho}, \partial_{j} u_{\rho}, \partial_{j, k}^{2} u_{\rho} \rightarrow u, \partial_{j} u, \partial_{j, k}^{2} u \quad \text { in } L^{p^{*}}\left(\mathbb{R}^{N}\right) \text { respectively, } \\
u_{\rho}, \partial_{j} u_{\rho}, \rightarrow u, \partial_{j} u, \quad \text { for almost every } x \in \mathbb{R}^{N} \text { respectively } \\
\partial_{j, k}^{2} u_{\rho} \rightarrow \partial_{j, k}^{2} u \quad \text { for almost every } x \in \mathbb{R}^{N}, \quad \text { if } p>2 .
\end{array}\right.
$$

For the proof of these properties, see $[1 ; \S 4$ in Chapter II]. Here we remark that in the sequel we can choose a subsequence on each occasion if necessary. Hence we can always assume that as $\rho \rightarrow 0, u_{\rho}, \partial_{j} u_{\rho}, \partial_{j, k}^{2} u_{\rho} \rightarrow u, \partial_{j} u, \partial_{j, k}^{2} u$ for almost every $x \in \mathbb{R}^{N}$ respectively.

Now we shall show that for every $u \in K_{p}\left(\mathbb{R}^{N}\right)$ having a compact support

$$
\mathcal{A}_{p} u_{\rho} \rightarrow \mathcal{A}_{p} u \quad \text { in } L^{1}\left(\mathbb{R}^{N}\right) \quad \text { as } \rho \rightarrow 0 .
$$

First it follows from the definition of the operator $\mathcal{A}_{p}$ that for a smooth function $v$

$$
\mathcal{A}_{p} v=\left(\operatorname{div}_{x} A\right)(x, \nabla v)+\sum_{j=1}^{N}\left(\partial_{\xi_{j}} A\right)(x, \nabla v) \cdot \nabla\left(\partial_{j} v\right) .
$$

Hence we have from (1.5) for some positive number $C$ independent of $v$

$$
\left|\mathcal{A}_{p} v\right| \leq C|\nabla v|^{p-2}\left(|\nabla v|+\left(\sum_{j, k=1}^{N} \partial_{j, k}^{2} v^{2}\right)^{\frac{1}{2}}\right) .
$$

When $p=2$, from (3.6) we immediately have

$$
\begin{aligned}
\mathcal{A}_{2} u_{\rho} & -\mathcal{A}_{2} u=\left(\left(\operatorname{div}_{x} A\right)\left(x, \nabla u_{\rho}\right)-\left(\operatorname{div}_{x} A\right)(x, \nabla u)\right) \\
& +\sum_{j=1}^{N}\left(\left(\partial_{\xi_{j}} A\right)\left(x, \nabla u_{\rho}\right) \cdot \nabla\left(\partial_{j} u_{\rho}\right)-\left(\partial_{\xi_{j}} A\right)(x, \nabla u) \cdot \nabla\left(\partial_{j} u\right)\right)
\end{aligned}
$$

We rewrite the first term in the right-hand side to obtain

$$
\left(\operatorname{div}_{x} A\right)\left(x, \omega_{\rho}\right)\left(\left|\nabla u_{\rho}\right|-|\nabla u|\right)+|\nabla u|\left(\left(\operatorname{div}_{x} A\right)\left(x, \omega_{\rho}\right)-\left(\operatorname{div}_{x} A\right)(x, \omega)\right) .
$$

Here $\omega_{\rho}$ and $\omega$ are given respectively by

$$
\omega_{\rho}=\left\{\begin{array}{l}
\frac{\nabla u_{\rho}}{\left|\nabla u_{\rho}\right|}, \text { if } \nabla u_{\rho} \neq 0 \\
0, \quad \text { if } \nabla u_{\rho}=0
\end{array} \quad \text { and } \quad \omega=\left\{\begin{array}{c}
\frac{\nabla u}{|\nabla u|}, \text { if } \nabla u \neq 0 \\
0, \quad \text { if } \nabla u=0
\end{array}\right.\right.
$$

From the homogeneity of $\operatorname{div}_{x} A,(3.4)$ and the remark just after it, we see these terms go to 0 in $L^{1}\left(\mathbb{R}^{N}\right)$ using the dominated convergence theorem. In a quite similar way we can show the convergence of the second term in $L^{1}\left(\mathbb{R}^{N}\right)$. 
Now we assume that $p>2$. Then from (3.7) and Young's inequality it holds that for any $\rho>0$

$$
\left|\mathcal{A}_{p} u_{\rho}\right| \leq C\left(\left|\nabla u_{\rho}\right|^{p-1}+\left(\sum_{j, k=1}^{N} \partial_{j, k}^{2} u_{\rho}^{2}\right)^{\frac{p-1}{2}}\right) .
$$

Here $C$ is a positive number independent of each $\rho>0$. If we show that $\mathcal{A}_{p} u_{\rho} \in L^{1}\left(\mathbb{R}^{N}\right)$ is dominated by an integrable function uniformly in $\rho>0$, then the assertion clearly follows again from the dominated convergence theorem.

For reader's convenience we shall explain this fact briefly. Since the argument is similar, we shall show that for every $u \in L^{q}\left(\mathbb{R}^{N}\right)$ having a compact support with $1<q<+\infty, u_{\rho} \in L^{q}\left(\mathbb{R}^{N}\right)$ can be dominated by some function in $L^{q}\left(\mathbb{R}^{N}\right)$ uniformly in $\rho>0$. Let us set $\tilde{u}_{\rho}(x)=\sup _{0<\rho}\left|u_{\rho}(x)\right|$. Then clearly $\left|u_{\rho}(x)\right| \leq\left|\tilde{u}_{\rho}(x)\right|$. Recall that $\varphi$ is radial and decreasing. Then from the argument $[1 ; \S 4$ in Chapter II] we can show $\left|\tilde{u}_{\rho}(x)\right| \leq C M u(x)$, where $M u(x)$ is the Hardy-Littlewood maximal function defined by $M u(x)=\sup _{x \in Q} \frac{1}{|Q|} \int_{Q}|u(y)| d y$, where the supremum is taken over all cubes $Q$ containing $x$, and $|Q|$ stands for the Lebesgue measure of $Q$. Then from the theory of the Hardy-Littlewood maximal function it follows that $M u \in L^{q}\left(\mathbb{R}^{N}\right)$. After all we see the desired property $\left|u_{\rho}\right| \leq C M u(x) \in L^{q}\left(\mathbb{R}^{N}\right)$, where $C$ is a positive number independent of each $\rho$.

Hence from (3.4), the remark just after it and the dominated convergence theorem we have $\mathcal{A}_{p} u_{\rho} \rightarrow \mathcal{A}_{p} u$ in $L^{1}\left(\mathbb{R}^{N}\right)$ as $\rho \rightarrow 0$.

From Lemma 2.1 and the dominated convergence theorem we see

$$
\begin{aligned}
\mathcal{A}_{p}\left(u_{\rho}\right)_{\varepsilon} \geq & \left|\frac{u_{\rho}}{\left(u_{\rho}\right)_{\varepsilon}}\right|^{p-2} \frac{u_{\rho}}{\left(u_{\rho}\right)_{\varepsilon}} \mathcal{A}_{p} u_{\rho} \\
= & \left|\frac{u_{\rho}}{\left(u_{\rho}\right)_{\varepsilon}}\right|^{p-2} \frac{u_{\rho}}{\left(u_{\rho}\right)_{\varepsilon}}\left(\mathcal{A}_{p} u_{\rho}-\mathcal{A}_{p} u\right) \\
& +\mathcal{A}_{p} u\left(\left|\frac{u_{\rho}}{\left(u_{\rho}\right)_{\varepsilon}}\right|^{p-2} \frac{u_{\rho}}{\left(u_{\rho}\right)_{\varepsilon}}-\left|\frac{u}{(u)_{\varepsilon}}\right|^{p-2} \frac{u}{(u)_{\varepsilon}}\right) \\
& +\left|\frac{u}{(u)_{\varepsilon}}\right|^{p-2} \frac{u}{(u)_{\varepsilon}} \mathcal{A}_{p} u \longrightarrow\left|\frac{u}{(u)_{\varepsilon}}\right|^{p-2} \frac{u}{(u)_{\varepsilon}} \mathcal{A}_{p} u, \quad \text { as } \rho \rightarrow 0 .
\end{aligned}
$$

Since $\mathcal{A}_{p}\left(u_{\rho}\right)_{\varepsilon} \rightarrow \mathcal{A}_{p} u_{\varepsilon}$ in the sense of the distribution, we get

$$
\mathcal{A}_{p} u_{\varepsilon} \geq\left|\frac{u}{u_{\varepsilon}}\right|^{p-2} \frac{u}{u_{\varepsilon}} \mathcal{A}_{p} u \quad \text { in } \mathcal{D}^{\prime}\left(\mathbb{R}^{N}\right) .
$$

Then by letting $\varepsilon \rightarrow 0$,we see $\mathcal{A}_{p} u_{\varepsilon} \rightarrow \mathcal{A}_{p}|u|$ in the sense of the distribution, and the right-hand side tends to $(\operatorname{sgn} u) \mathcal{A}_{p} u$ in $L_{\text {loc }}^{1}\left(\mathbb{R}^{N}\right)$. Therefore we get the desired inequality. 
We proceed to the case that $1<p<2$. In this case we make use of $\mathcal{A}_{p, \eta}$ instead. Again it suffices to assume that $\Omega=\mathbb{R}^{N}$ and $u$ has a compact support. In a quite similar way, we see for any $\eta>0$

$$
\begin{aligned}
& \left|\mathcal{A}_{p, \eta} u\right| \\
\leq & \left|\nabla_{x} a\left(x,|\nabla u|_{\eta}\right)\right||\nabla u|+\sum_{j, k=1}^{N}\left|\partial_{j, k}^{2} u\right|\left(a\left(x,|\nabla u|_{\eta}\right)+\frac{\partial}{\partial r} a\left(x,|\nabla u|_{\eta}\right)|\nabla u|_{\eta}\right) \\
\leq & C|\nabla u|_{\eta}^{p-2}\left(|\nabla u|+\sum_{j, k=1}^{N}\left|\partial_{j, k}^{2} u\right|\right),
\end{aligned}
$$

where $|\nabla u|_{\eta}=\left(\eta^{2}+|\nabla u|^{2}\right)^{\frac{1}{2}}$. Since $|\nabla u|_{\eta}^{p-2}$ is bounded for $\eta>0$, we see $\mathcal{A}_{p, \eta} u \in$ $L_{\text {loc }}^{1}\left(\mathbb{R}^{N}\right)$. Here we note that $1<p<2$ and $\partial_{j, k}^{2} u \in L^{1}\left(\mathbb{R}^{N}\right)$ for $j, k=1,2, \cdots, N$. Let $u_{\rho}$ be defined by (3.3). Then it follows from Lemma 2.2 that $\left(u_{\rho}\right)_{\varepsilon}$ satisfies

$$
\begin{aligned}
& \mathcal{A}_{p, \eta}\left(u_{\rho}\right)_{\varepsilon} \\
\geq & \frac{u_{\rho}}{\left(u_{\rho}\right)_{\varepsilon}}\left(a\left(x,\left|\nabla\left(u_{\rho}\right)_{\varepsilon}\right|_{\eta}\right) \Delta u_{\rho}+\nabla u_{\rho} \cdot \nabla_{x} a\left(x,\left|\nabla\left(u_{\rho}\right)_{\varepsilon}\right|_{\eta}\right)\right. \\
& \left.+\frac{\partial}{\partial r} a\left(x,\left|\nabla\left(u_{\rho}\right)_{\varepsilon}\right|_{\eta}\right)\left|\nabla\left(u_{\rho}\right)_{\varepsilon}\right|_{\eta}\left(\frac{u_{\rho}}{\left(u_{\rho}\right)_{\varepsilon}}\right)^{2} \sum_{j, k} \frac{\partial_{j} u_{\rho} \partial_{k} u_{\rho} \partial_{j, k}^{2} u_{\rho}}{\left|\nabla\left(u_{\rho}\right)_{\varepsilon}\right|_{\eta}^{2}}\right)
\end{aligned}
$$

As $\rho \rightarrow 0$, we see $\mathcal{A}_{p, \eta}\left(u_{\rho}\right)_{\varepsilon} \rightarrow \mathcal{A}_{p, \eta}(u)_{\varepsilon}$ in the sense of distribution, and the terms in the right-hand side also converges in $L^{1}\left(\mathbb{R}^{n}\right)$. Here we note that $a\left(x,\left|\nabla\left(u_{\rho}\right)_{\varepsilon}\right|_{\eta}\right), \nabla_{x} a\left(x,\left|\nabla\left(u_{\rho}\right)_{\varepsilon}\right|_{\eta}\right)$ and $\frac{\partial}{\partial r} a\left(x,\left|\nabla\left(u_{\rho}\right)_{\varepsilon}\right|_{\eta}\right)\left|\nabla\left(u_{\rho}\right)_{\varepsilon}\right|_{\eta}$ are uniformly bounded in $\rho$ by the homogeneity and positivity of $\eta$. Therefore we get in $\mathcal{D}^{\prime}\left(\mathbb{R}^{N}\right)$

$$
\begin{aligned}
\mathcal{A}_{p, \eta} u_{\varepsilon} \geq & \frac{u}{u_{\varepsilon}}\left(a\left(x,\left|\nabla u_{\varepsilon}\right|_{\eta}\right) \Delta u+\nabla u \cdot \nabla_{x} a\left(x,\left|\nabla u_{\varepsilon}\right|_{\eta}\right)\right. \\
& \left.+\frac{\partial}{\partial r} a\left(x,\left|\nabla u_{\varepsilon}\right|_{\eta}\right)\left|\nabla u_{\varepsilon}\right|_{\eta}\left(\frac{u}{u_{\varepsilon}}\right)^{2} \sum_{j, k} \frac{\partial_{j} u \partial_{k} u \partial_{j, k}^{2} u}{\left|\nabla u_{\varepsilon}\right|_{\eta}^{2}}\right)
\end{aligned}
$$

Letting $\varepsilon \rightarrow 0$ we have in a similar way in $\mathcal{D}^{\prime}\left(\mathbb{R}^{N}\right)$

$$
\begin{aligned}
\mathcal{A}_{p, \eta}|u| \geq & (\operatorname{sgn} u)\left(a\left(x,|\nabla u|_{\eta}\right) \Delta u+\nabla u \cdot \nabla_{x} a\left(x,|\nabla u|_{\eta}\right)\right. \\
& \left.+\frac{\partial}{\partial r} a\left(x,|\nabla u|_{\eta}\right)|\nabla u|_{\eta} \sum_{j, k} \frac{\partial_{j} u \partial_{k} u \partial_{j, k}^{2} u}{|\nabla u|_{\eta}^{2}}\right) \\
= & (\operatorname{sgn} u) \mathcal{A}_{p, \eta} u
\end{aligned}
$$

Finally by letting $\eta \rightarrow 0$, we have in the sense of distribution $\mathcal{A}_{p, \eta}|u| \rightarrow \mathcal{A}_{p}|u|$, and the right-hand side also converges in $L_{l o c}^{1}\left(\mathbb{R}^{N}\right)$. After all we get

$$
\mathcal{A}_{p}|u| \geq(\operatorname{sgn} u) \mathcal{A}_{p} u \quad \text { in } \mathcal{D}^{\prime}\left(\mathbb{R}^{N}\right) .
$$


In a similar way we can show

$$
\mathcal{A}_{p} u_{+} \geq\left(\operatorname{sgn}^{+} u\right)^{p-1} \mathcal{A}_{p} u \quad \text { in } \mathcal{D}^{\prime}\left(\mathbb{R}^{N}\right),
$$

by making use of Lemma 2.2. Therefore the assertions are proved.

\section{References}

[1] J. Garcia-Cuerva and J. L. Rubio de Francia, Weighted norm inequalities and related Topics, North-Holland Mthematics Studies, 116(1985).

[2] T. Horiuchi, Some remarks on Kato's inequality, J. of Inequalities and applications., 6(2001), 29-36.

[3] T. Kato, Schrödinger operators with singular potentials, Israel J. Math., 13(1972), 135-148. 